\title{
Galactose-Modified Cationic Liposomes as a Liver-Targeting Delivery System for Small Interfering RNA
}

\author{
Satoru Sonoke, * Toshihiro Ueda, Kae Fujiwara, Kenji Kuwabara, and Junichi Yano \\ Discovery Research Laboratories, Nippon Shinyaku Co., Ltd.; 3-14-1 Sakura, Tsukuba, Ibaraki 305-0003, Japan.
}

Received December 16, 2010; accepted May 7, 2011; published online May 27, 2011

\begin{abstract}
We have developed a galactose-modified cationic liposome for delivery of small interfering RNA (siRNA) to the liver. The liposomes were designed to be transported into hepatocytes via the asialoglycoprotein receptor, which recognizes galactose residues. The liposomes contained a novel galactose-modified lipid, 1,2-dioleoyl-snglycerol-3-phosphatidyl- $N$-(1-deoxylactito-1-yl)ethanolamine (GDOPE). Delivery of siRNA to hepatocytes by the liposomes was evaluated by measuring the gene-silencing activity of liposome : siRNA complexes in two human hepatoma cell lines. A formulation with a cationic lipid: GDOPE ratio of 3:5 by weight, LIC-G5, showed the strongest activity. In mice, intravenous injection of LIC-G5 complexed with ${ }^{3} \mathrm{H}$-labeled siRNA led to accumulation of radioactivity in the liver. When the hepatic cellular uptake was determined after intravenous injection into mice followed by collagenase liver perfusion, the distribution of siRNA to parenchymal cells was 1.9 times higher when LIC-G5 rather than nongalactosylated LIC was used as the carrier. The concentration of siRNA accumulated was $45 \mu \mathrm{g} / \mathrm{ml}, 30$ times the concentration that produced strong gene silencing in vitro and therefore presumably sufficient for a therapeutic effect. Because increasing the cationic-lipid content of a liposome carrier generally enhances the uptake of siRNA by the liver at the expense of increased cell toxicity, we used only a moderate amount of cationic lipid in our galactose-modified carrier. LIC-G5 enhanced the uptake of siRNA by the liver without cytotoxic effects and is a promising candidate delivery system for liver-targeted siRNA therapy.
\end{abstract}

Key words small interfering RNA; cationic liposome; hepatocyte; galactose; drug delivery

Sequence-specific gene silencing based on RNA interference (RNAi) ${ }^{1-3)}$ holds great promise for molecular-targeted treatment of diseases. RNAi is mediated by small interfering RNA (siRNA), which is under development as a new type of therapeutic agent. siRNA is easily synthesized and highly specific and its pharmacokinetics are expected to be largely independent of sequence. In most clinical studies of siRNA, chemically modified siRNA without a carrier has been administered locally, for example to the eye for age-related macular degeneration or to the lung for respiratory syncytial virus infection. ${ }^{4-6)}$ However, perhaps because of the absence of a clear gene-silencing effect in most clinical trials, the development of siRNA as a therapeutic agent has not been advancing rapidly. Although systemic administration is desirable for the treatment of diseases such as cancer and viral infections, it has not yet been validated for siRNA in clinical trials.

Receptor-mediated drug targeting is a promising approach to delivering drugs to specific cell types. ${ }^{7)}$ For example, on the surface of hepatocytes there is a receptor that recognizes the galactose residue of asialoglycoproteins and mediates their uptake. ${ }^{8)}$ In the present study, we designed a cationic liposome containing a phospholipid modified with a galactose residue for delivery of siRNA to the liver. This novel phospholipid derivative is 1,2-dioleoyl-sn-glycerol-3-phosphatidyl$N$-(1-deoxylactito-1-yl)ethanolamine (GDOPE; Fig. 1). When complexed with liposomes containing this galactosylated phospholipid, siRNA showed specific gene silencing in vitro and enhanced transfer to hepatocytes in vivo.

\section{MATERIALS AND METHODS}

Materials 1-Palmitoyl-2-oleoyl-sn-glycero-3-phosphocholine (POPC) was purchased from NOF (Tokyo, Japan) and $\left[2,5^{\prime}, 8-{ }^{3} \mathrm{H}\right]$ adenosine $5^{\prime}$-triphosphate, ammonium salt, galactose-modified lipid (GDOPE)

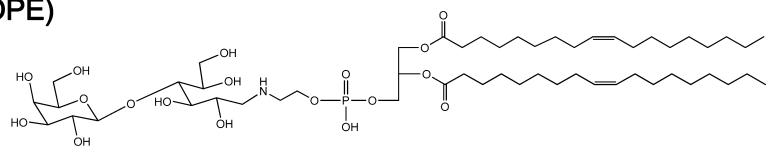

cationic lipid (CLZ-42)

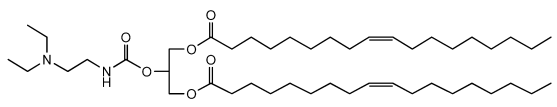

PEG-lipid

helper lipid

(POPC)
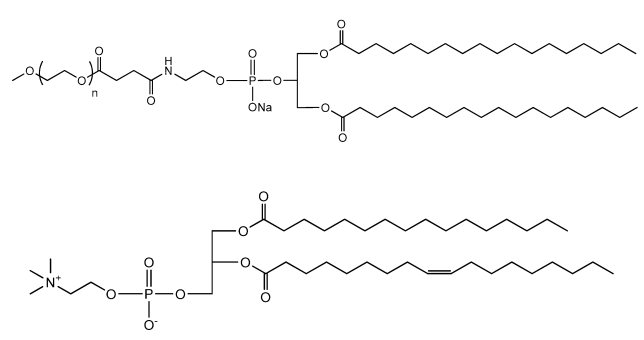

Fig. 1. Chemical Structures of Liposome Components

from Mandel NEN Products (Guelph, ON, Canada). All other chemicals were of analytical or reagent grade and were used without further purification.

Animals Male Balb/c mice aged 4-6 weeks were purchased from Clea Japan (Tokyo, Japan) and housed in a room maintained at $21-25^{\circ} \mathrm{C}$ and a humidity of $50-60 \%$. Animal experiments were carried out in accordance with Nippon Shinyaku Guidelines for the Care and Use of Laboratory Animals.

Synthesis of Lipids GDOPE was synthesized as follows. Equimolar amounts of lactose and 1,2-dioleoyl-snglycero-3-phosphatidylethanolamine were suspended in dichloromethane/methanol/water/acetic acid (2:8:2:1, $\mathrm{v} / \mathrm{v} / \mathrm{v} / \mathrm{v}$ ). Sodium cyanoborohydride ( $3 \mathrm{eq}$ ) was added and the 
reaction mixture was heated at $70^{\circ} \mathrm{C}$ for $16 \mathrm{~h}$. The reaction mixture was then evaporated almost to dryness and the residue was partitioned between dichloromethane and water. The aqueous layer was extracted with dichloromethane and the combined organic extracts were washed with water and dried over anhydrous magnesium sulfate. The organic solvent was removed under reduced pressure and the residue was purified by flash chromatography with dichloromethane/ methanol/water $(60: 20: 3, \mathrm{v} / \mathrm{v} / \mathrm{v})$ as the solvent to afford GDOPE as a white powder. The cationic lipid 2-O-(2-diethylaminoethyl)carbamoyl-1,3-O-dioleoylglycerol (CLZ-42) was synthesized as described previously.,10) Each synthesized lipid was isolated as a single component on TLC, gave a mass spectrum consistent with the expected structure, and gave the expected characteristic ${ }^{1} \mathrm{H}-\mathrm{NMR}$ signals (data not shown).

Synthesis of siRNA A model siRNA, siLuc, which is sequence-specific for firefly luciferase mRNA, was synthesized on an Expedite 8909 DNA synthesizer (Applied Biosystems, Foster City, CA, U.S.A.). Its purity was $>90 \%$ as determined by capillary gel electrophoresis. The small interfering RNA for vascular endothelial growth factor siVEGF, which is sequence-specific for human vascular endothelial growth factor A (hVEGF-A) mRNA, was purchased from Ambion (Austin, TX, U.S.A.). The siRNA sequences were: siLuc sense strand, 5'-GCUAUGAAACGAUAUGGGCdTdT-3'; antisense strand, 5'-GCCCAUAUCGUUUCAUAGCdTdT-3'; siVEGF sense strand, 5'-GGAGUACCCUGAUGAGAUCdTdT-3'; antisense strand, 5'-GAUCUCAUCAGGGUACUCCdTdT-3'.

Enzymatic Synthesis of ${ }^{3} \mathbf{H}$-Labeled siRNA Tritiumlabeled siLuc was prepared from ${ }^{3} \mathrm{H}$-labeled $\left[2,5^{\prime}, 8-{ }^{3} \mathrm{H}\right]$ adenosine 5 '-triphosphate and unlabeled deoxyribonucleoside triphosphates by using T7 RNA polymerase with the in vitro Transcription T7 Kit from Takara (Kyoto, Japan). The DNA template was synthesized by Hokkaido Systems Science (Sapporo, Hokkaido, Japan). The specific activity of the labeled siLuc was $6.4 \times 10^{5} \mathrm{dpm} / \mu \mathrm{g}$.

Preparation and Characterization of Cationic-Liposome : siRNA Complexes LIC complexed with siRNA was prepared as previously described. ${ }^{9)}$ LIC-G complexes containing the cationic lipid CLZ-42, POPC as a helper lipid, GDOPE and siRNA were prepared by homogenization and emulsification in $10 \%(\mathrm{w} / \mathrm{v})$ maltose with a Branson Sonifier Model 250D (Branson Ultrasonics, Danbury, CT, U.S.A.). The lipid composition of the complexes is shown in Table 1. The particle-size distributions of the LIC-G complexes were determined by dynamic laser light scattering with a Nicomp 380 particle sizer (Particle Sizing Systems, Santa Barbara, CA, U.S.A.). Volume-weighted Gaussian distribution analysis was used to determine the mean particle diameter. For transmission electron microscopy (TEM), samples were placed on a specimen mesh coated with collodion film, negatively stained with sodium phosphotungstate, $\mathrm{pH}$ 7.0, and observed under a JEM1200EX electron microscope (JEOL, Tokyo, Japan) operated at $100 \mathrm{kV}$.

Cellular Uptake of siRNA in Vitro Huh-7 cells (a human hepatoma cell line) were seeded on a 6 -well plate at $2.5 \times 10^{5}$ cells/well and incubated for $18 \mathrm{~h}$ at $37^{\circ} \mathrm{C}$ under $5 \%$ $\mathrm{CO}_{2}$. The medium was changed and LIC-G complex containing ${ }^{3} \mathrm{H}$-labeled siLuc was added. After $8 \mathrm{~h}$, the cells were detached by treatment with trypsin-ethylenediaminetetraacetic
Table 1. Lipid Composition and Particle Sizes of Cationic-Liposome Formulations

\begin{tabular}{crrrrr}
\hline \hline & \multicolumn{4}{c}{ Components (relative weight) } & $\begin{array}{c}\text { Mean particle } \\
\text { size (nm) }\end{array}$ \\
\cline { 2 - 5 } & CLZ-42 & POPC & GDOPE & siRNA & \\
\cline { 2 - 5 }$\# 1$ & 5 & 4 & 6 & 1 & $139 \pm 8$ \\
$\# 2$ & 6 & 4 & 6 & 1 & $136 \pm 3$ \\
$\# 3$ & 7 & 4 & 6 & 1 & $132 \pm 10$ \\
$\# 4$ & 8 & 4 & 6 & 1 & $119 \pm 7$ \\
$\# 5$ & 9 & 4 & 6 & 1 & $116 \pm 9$ \\
$\# 6$ & 10 & 4 & 6 & 1 & $121 \pm 1$ \\
$\# 7$ & 11 & 4 & 6 & 1 & $114 \pm 7$ \\
$\# 8$ & 12 & 4 & 6 & 1 & $108 \pm 2$ \\
LIC-G1 & 6 & 8 & 2 & 1 & $148 \pm 2$ \\
LIC-G2 & 6 & 6 & 4 & 1 & $154 \pm 2$ \\
LIC-G3 & 6 & 4 & 6 & 1 & $136 \pm 3$ \\
LIC-G4 & 6 & 2 & 8 & 1 & $149 \pm 12$ \\
LIC-G5 & 6 & 0 & 10 & 1 & $147 \pm 12$ \\
LIC & 6 & 10 & 0 & 1 & $142 \pm 3$ \\
\hline
\end{tabular}

a) Data are the mean \pm S.D. for at least three different preparations.

acid (EDTA) solution (Sigma, St. Louis, MO, U.S.A.), collected by centrifugation, and dissolved in Hionic-Fluor scintillation cocktail (PerkinElmer, Waltham, MA, U.S.A.), and the radioactivity was measured in a Tri-Carb 2700TR liquid scintillation counter (Packard, Meriden, CT, U.S.A.).

Assessment of Cell Viability Cell viability was assessed by tetrazolium dye metabolic assay by measuring the mitochondrial reduction of WST- 8 to the water-soluble formazan. Measurements were made with Cell Counting Kit-8 (Dojindo, Kumamoto, Japan). Huh- 7 cells $\left(5 \times 10^{3}\right.$ cells $\left./ \mathrm{cm}^{2}\right)$ or HepG2 cells (another human hepatoma cell line; $1 \times$ $10^{4}$ cells $/ \mathrm{cm}^{2}$ ) were seeded on 24-well plates and incubated for $48 \mathrm{~h}$. LIC-G complex was added and incubation continued for a further $48 \mathrm{~h}$. The cells were then incubated with WST- 8 assay solution for $1 \mathrm{~h}$ and the absorbance of the formazan dye generated was measured at $450 \mathrm{~nm}$. The absorbance values were used to calculate cell survival relative to that of untreated control cells.

Assessment of Gene Silencing Huh-7 or HepG2 cells were seeded on a 12 -well plate at 1 or $2 \times 10^{5}$ cells/well, respectively, and incubated for $18 \mathrm{~h}$ at $37^{\circ} \mathrm{C}$ under $5 \% \mathrm{CO}_{2}$. The next day, the culture medium was changed, LIC$\mathrm{G}$ : siVEGF was added, and incubation was continued for a further $24 \mathrm{~h}$ under the same conditions. The amount of hVEGF-A protein in the culture supernatant was then measured with a human VEGF immunoassay kit (R\&D Systems, Minneapolis, MN, U.S.A.). Control cells were treated with $10 \%$ maltose.

Hepatic Cellular Uptake of siRNA in Vivo LIC-G complex containing ${ }^{3} \mathrm{H}$-labeled siLuc was administered to mice via the tail vein at a dose of $10 \mathrm{mg} / \mathrm{kg}$. After $1 \mathrm{~h}$, the mice were laparotomized and the liver was perfused in an open circuit through the portal vena cava and the aorta. Perfusion was maintained for $10 \mathrm{~min}$ with $0.1 \mathrm{M} N$-(2-hydroxyethyl)piperazine- $N^{\prime}$-2-ethanesulfonic acid (HEPES) buffer, $\mathrm{pH} 7.5$, and then for $15 \mathrm{~min}$ with the same buffer containing $0.05 \%$ collagenase. The liver was then removed and the parenchymal cells (PC; i.e., hepatocytes) and non-parenchymal cells (NPC) were separated by differential centrifugation. ${ }^{11)}$ The final 50-g (PC) and 600-g (NPC) pellets were re- 
suspended in phosphate-buffered saline and homogenized, and their radioactivity was determined by scintillation counting.

Statistical Analysis The data were analyzed by Dunnett's multiple-comparison test with the software package SAS Version 8.2 (SAS Institute, Cary, NC, U.S.A.). A $p$ value of $<0.01^{* *}$ or $<0.05^{*}$ was taken to indicate a statistically significant difference.

\section{RESULTS AND DISCUSSION}

We optimized the cationic-lipid content of LIC-G by measuring the particle-size distribution and toxicity towards HepG2 and Huh-7 cells of formulations with increasing cationic-lipid content complexed with siRNA (siVEGF). The mean particle diameter of the LIC-G complexes decreased slightly with increasing cationic-lipid content (Table 1). Although cell viability was not affected by the cationic-lipid content of the complexes at $10 \mathrm{~nm}$ siVEGF, the viability of HepG2 cells (but not Huh-7 cells) decreased slightly with in-

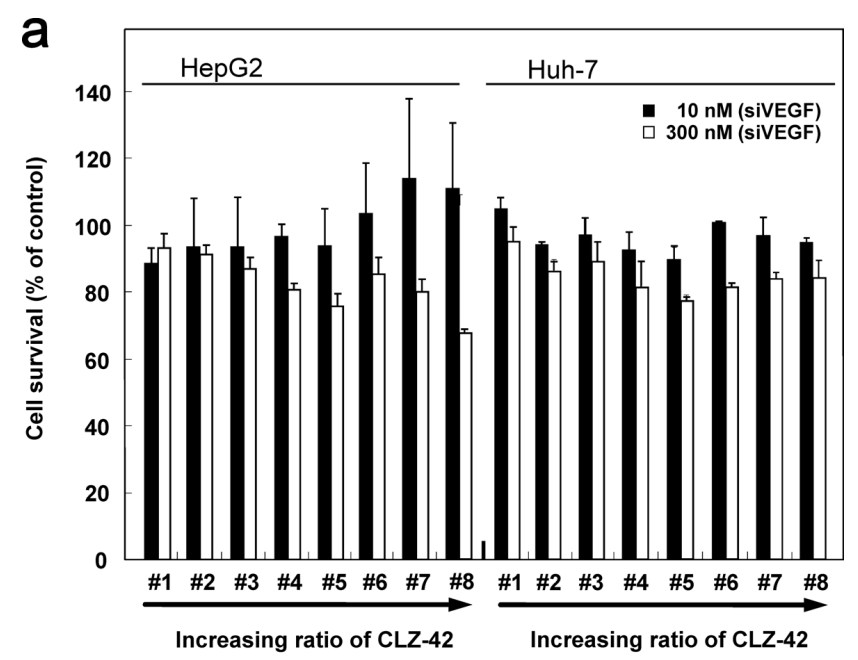

b

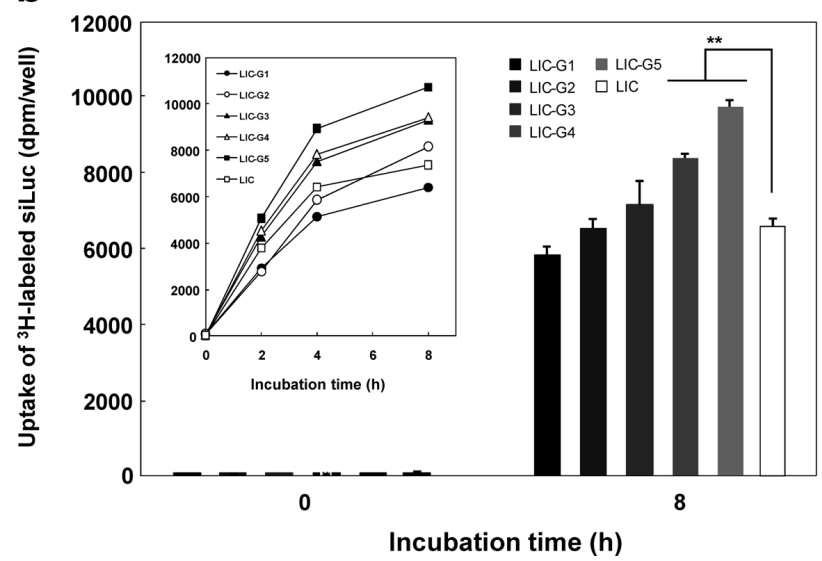

Fig. 2. Effect of Lipid Components of LIC-G Complexes on Cell Survival and siRNA Uptake

(a) Cell survival as a function of cationic-lipid content. The survival of HepG2 and Huh-7 cells relative to control cells treated with $10 \%$ maltose is expressed as the mean \pm S.D. $(n=3)$. (b) Uptake of siRNA complexed with LIC-G formulations with increasing GDOPE content. Tritium-labeled siLuc complexed with each formulation was incubated with Huh-7 cells, and at the indicated times cells were collected and the radioactivity was measured. Bars represent the mean \pm S.D. $(n=3) * * p<0.01$. Inset, time courses. creasing cationic-lipid content at $300 \mathrm{~nm}$ siVEGF (Fig. 2a). Because of this slight effect at the higher concentration, we used LIC-G with the relatively low cationic-noncationic lipid ratio of $6: 10$ for further investigation. In these complexes, the ratio of siRNA to total lipid was $1: 16(\mathrm{w} / \mathrm{w})$.

We next determined the relative transfection efficiency of LIC-G1-LIC-G5, a series of formulations with increasing GDOPE content (Table 1), complexed with ${ }^{3} \mathrm{H}$-labeled siLuc by measuring the radioactivity taken up by Huh-7 cells during an 8-h incubation with the complexes (Fig. 2b). Radioactivity was taken up by the cells rapidly at first and more slowly towards the end of the incubation period. At each time point, the uptake tended to increase with increasing GDOPE content.

We then investigated the gene-silencing activity in Huh-7 cells of LIC-G1, LIC-G3 and LIC-G5 complexed with siVEGF. The formulations decreased the production of hVEGF-A protein by $30-90 \%$ (Fig. 3a, left panel). In particular, the LIC-G5 complex produced about twofold greater suppression of hVEGF-A than did the nongalactosylated LIC complex. Furthermore, the gene-silencing effect of the LICG5 complex was dose-dependent (Fig. 3a, right panel). Because LIC-G3 had no noticeable effect on cell viability (Fig.
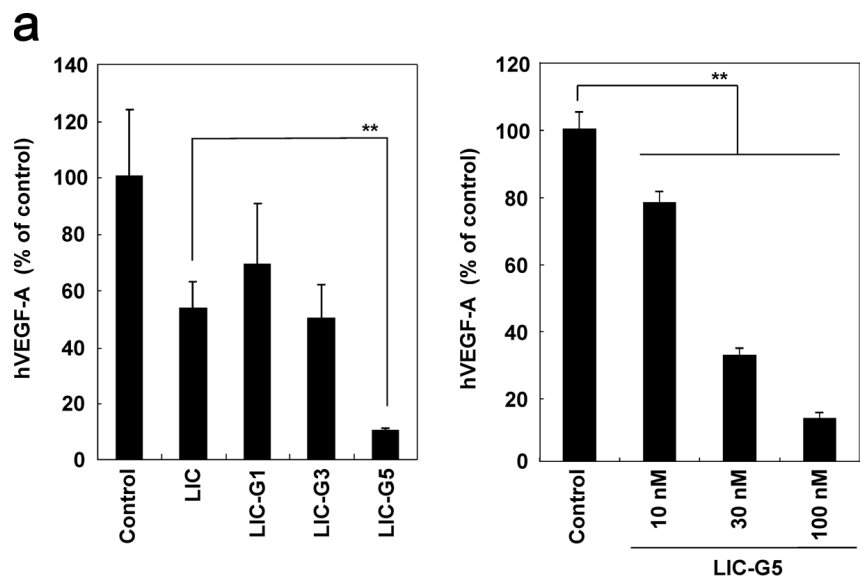

b
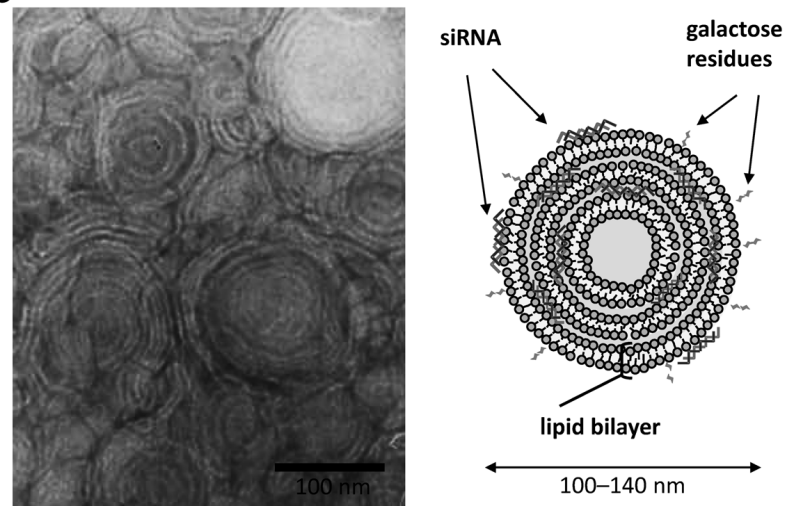

Fig. 3. Gene-Silencing Activity and Morphology of LIC-G : siRNA Complexes

(a) Gene silencing in Huh-7 cells of three LIC-G formulations (100 nm siRNA; left panel) and three concentrations of LIC-G5 (right panel) complexed with siVEGF. After incubation of the cells with each formulation, the amount of hVEGF-A protein in the culture supernatant was determined and calculated relative to that produced by control cells treated with $10 \%$ maltose. The values are expressed as the mean \pm S.D. $(n=3)$ $* * p<0.01$. (b) Left panel, TEM micrograph of LIC-G5 complexed with siVEGF. Right panel, schematic drawing of LIC-G complexed with siRNA. 
2a), we were not concerned that the complexes LIC-G1LIC-G5 tested in the transfection or gene-silencing experiments would have toxic effects on the cells (all of these complexes had a cationic: noncationic lipid ratio of $6: 10$; Table 1). LIC-G3 gave a weaker gene-silencing effect (Fig. 3a, left panel) than might have been expected from the extent of its uptake (Fig. 2b). It is likely that siRNA : LIC-G is taken up by endocytosis, ${ }^{12}$ and release from the endosomes to the cytosol is necessary for gene silencing. However, the rate of endosomal escape may vary with the lipid composition, and there may not be a simple linear relationship between the extent of uptake and the gene-silencing effect for formulations with different lipid compositions.

LIC-G5 is the only liposome in this study that contained no POPC helper lipid. (A helper lipid is a neutral lipid used in cationic liposomes complexed with nucleic acids to mediate fusion between the liposomes and the endosomal membrane after cellular uptake of the complexes, thus promoting release of nucleic acid from the endosomes. ${ }^{13)}$ ) Because RNAi takes place in the cytosol, the fact that the LIC-G5 complex, which contains no POPC, showed the strongest gene silencing of any of the complexes tested indicates that the addition of a helper lipid was not necessary for endosomal release of the siRNA in this cationic lipid system. Previous work on the design of helper lipids for use with cationic liposomes for siRNA delivery ${ }^{14}$ ) suggests that our novel galactosylated phospholipid derivative, GDOPE, may itself function as a helper lipid in this system.

By TEM with negative staining, the LIC-G5:siVEGF complex was visualized as multilamellar vesicles (Fig. 3b, left panel). The dark-staining aqueous regions correspond to the condensed siRNA, ${ }^{15)}$ while the light, unstained regions correspond to the lipid components (see Fig. 3b, right panel). The sizes of the vesicles observed by TEM are in reasonable agreement with the mean particle size of $147 \mathrm{~nm}$ determined by laser particle size analysis. siRNA probably becomes trapped between the lipid bilayers and is protected from enzymatic degradation, as we have shown for LIC. ${ }^{10)}$ The galactose residues exposed on the outer surface of the liposomes are intended to promote uptake of the complex into hepatocytes by the asialoglycoprotein receptor (ASGR).

The plasma and liver distribution of radioactivity was investigated after administration of cationic liposome:siLuc complexes containing ${ }^{3} \mathrm{H}$-labeled siLuc to mice by a single injection into the tail vein at a dose of $5 \mathrm{mg} / \mathrm{kg}$. Five minutes after administration, the liver accumulation of siLuc complexed with LIC-G5 was 1.7-fold higher than that of siLuc complexed with LIC, and after $60 \mathrm{~min}$ it was 1.5 -fold higher (Fig. 4a). Conversely, after $5 \mathrm{~min}$, the amount of siLuc remaining in the plasma when LIC-G5 was used as the carrier was $52 \%$ lower than when LIC was the carrier, and after 60 min it was $73 \%$ lower (Fig. 4a). With LIC-G5 as the carrier, siLuc was concentrated 2.9 -fold in the liver relative to the plasma $5 \mathrm{~min}$ after administration, whereas with LIC as the carrier siLuc was not concentrated in the liver.

When mice were pretreated with the ASGR ligand asialofetuin $1 \mathrm{~min}$ before injection of LIC-G5, the liver concentrations of siLuc fell to the levels obtained with the nongalactosylated carrier LIC (Fig. 4b, left panel). This provides evidence that the improved liver uptake of siLuc obtained with LIC-G5 was due to the ASGR. Thus, delivery of siRNA a

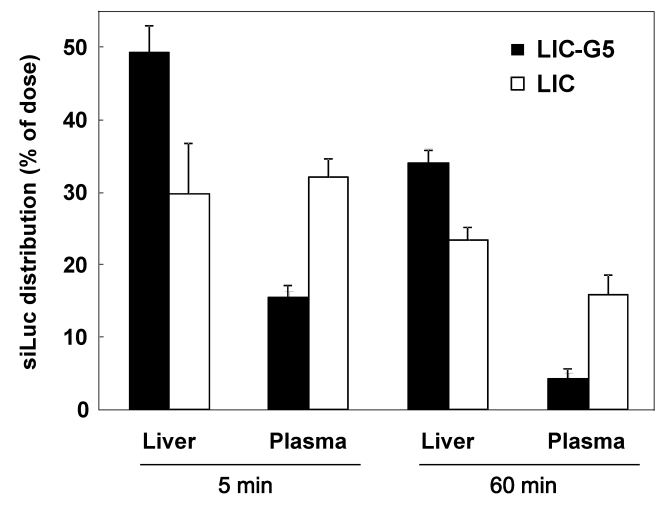

b
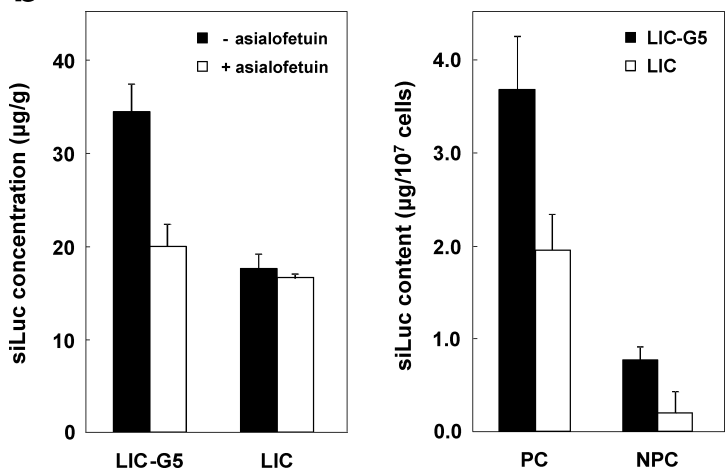

Fig. 4. Liver Distribution in Mice of siRNA Delivered by LIC-G5 or LIC

Mice were administered LIC-G5 or LIC complexed with ${ }^{3} \mathrm{H}$-labeled siLuc via the tail vein. (a) After administration of the complex at a dose of $5 \mathrm{mg} / \mathrm{kg}$, the plasma and liver content of radioactivity was measured. (b) Left panel, effect of asialofetuin pretreatment on liver concentration of siLuc. One minute after administration of asialofetuin $(10 \mathrm{mg} / \mathrm{kg})$ via the tail vein, labeled LIC-G5 : siLuc complex was administered at a dose of $5 \mathrm{mg} / \mathrm{kg}$ and the liver content of radioactivity was measured $60 \mathrm{~min}$ after administration. Right panel, 60 min after administration of labeled LIC-G5 : siLuc complex at a dose of $5 \mathrm{mg} / \mathrm{kg}$, collagenase liver perfusion was performed and the parenchymal cells (PC) and non-parenchymal cells (NPC) were isolated and their radioactivity was measured. In both experiments, siLuc concentrations are expressed as the mean \pm S.D. $(n=3)$.

to hepatocytes by LIC-G5 depended not only on nonspecific electrostatic adhesion of the liposomes to the cells but also on specific interaction of the exposed galactose residues with a cell-surface receptor.

To investigate the transfer of siRNA into hepatocytes by LIC-G5 in vivo, we administered LIC-G5 complexed with ${ }^{3} \mathrm{H}$-labeled siLuc into the tail vein of mice, performed collagenase liver perfusion, and isolated PC and NPC. The accumulation of siLuc in the PC was $3.7 \mu \mathrm{g} / 10^{7}$ cells, and in the NPC it was $0.8 \mu \mathrm{g} / 10^{7}$ cells (Fig. $4 \mathrm{~b}$, right panel). With LIC as the carrier, the accumulation of siLuc was lower in both PC and NPC, at 2.0 and $0.2 \mu \mathrm{g}$, respectively, per $10^{7}$ cells. Thus, the accumulation of siLuc in PC and NPC was 1.9 and 4.0 times higher, respectively, with LIC-G5 than with LIC. This suggests that LIC delivered siRNA more selectively to PC than LIC-G5 did (Fig. 4b, right panel), an observation that may be explained by the presence of a galactose-binding glycoprotein receptor on Kupffer cells, the most abundant type of NPC. ${ }^{16)}$ Because the particle sizes of LIC and LICG5 are almost the same (Table 1), the more selective uptake of LIC-G5 by NPC cannot be explained by the tendency of Kupffer cells to phagocytose large foreign particles more ef- 
fectively than small ones. However, when it is considered that NPC account for only about $20 \%$ of the total liver volume, our results show that most of the siRNA accumulated to the PC with either carrier. The point that we would like to emphasize here is that LIC-G5 produced an almost twofold greater accumulation of siRNA to PC than LIC did.

Several reports have described the use of liposomes targeting cell-surface galactosyl receptors to mediate nucleic acid delivery. Thus, Sato et al. ${ }^{17)}$ used cationic liposomes containing a galactosylated cholesterol derivative to deliver siRNA to the liver. They observed enhanced cellular accumulation of fluorescein-labeled siRNA with the galactosylated liposomes in liver sections and suggested that the siRNA accumulated in the PC. A similar study by Wang et al. ${ }^{18)}$ investigated neutral liposomes containing a cholesterol derivative with galactosylated polymers for delivery of oligodeoxynucleotides to the liver. They measured enhanced accumulation of fluorescently labeled liposome complex with the galactosylated liposomes in liver sections. Although these studies have demonstrated liver targeting of oligonucleotides by liposomes bearing galactosyl residues, as far as we know our study is the first to quantify the accumulation of oligonucleotides in isolated PC with ${ }^{3} \mathrm{H}$-labeled siRNA. In addition, the galactosylated component of our liposomes, GDOPE, is a novel galactosylated phospholipid derivative, in contrast to the galactosylated cholesterol derivatives previously used.

Sixty minutes after administration, the concentration of siLuc in PC with LIC-G5 as the carrier was $3.7 \mu \mathrm{g} / 10^{7}$ cells (about $2.8 \mu \mathrm{M}$ ), almost 30 times the concentration of $100 \mathrm{nM}$ siVEGF that gave strong gene silencing in Huh-7 cells in vitro (Fig. 3a, right panel). Therefore the concentration of siRNA in the PC achieved by LIC-G5 is in principle sufficient for a therapeutic effect. In addition, we have shown that galactose-modified liposomes based on LIC could achieve enhanced liver concentrations of siRNA without any increase in cationic-lipid content. This is an advantage because a high cationic-lipid content was associated with slight cell toxicity at a high concentration. LIC-G5 showed improved delivery of siRNA both into hepatocytes in vitro and into the liver in vivo, as well as improved gene silencing, compared to LIC despite having no POPC helper lipid. In conclusion, LIC-G5 is a promising liver-targeting delivery system for siRNA which may also be developed for the delivery of other RNA molecules of therapeutic interest.
Acknowledgement We thank Dr. Gerald E. Smyth, Discovery Research Laboratories, Nippon Shinyaku Co., Ltd., for helpful discussions, suggestions and support during the preparation of the manuscript.

\section{REFERENCES}

1) Fire A., Xu S., Montgomery M. K., Kostas S. A., Driver S. E., Mello C. C., Nature (London), 391, 806-811 (1998).

2) Elbashir S. M., Harborth J., Lendeckel W., Yalcin A., Weber K., Tuschl T., Nature (London), 411, 494-498 (2001).

3) Novina C. D., Sharp P. A., Nature (London), 430, 161-164 (2004).

4) Shen J., Samul R., Silva R. L., Akiyama H., Liu H., Saishin Y., Hackett S. F., Zinnen S., Kossen K., Fosnaugh K., Vargeese C., Gomez A., Bouhana K., Aitchison R., Pavco P., Campochiaro P. A., Gene Ther. 13, 225-234 (2006).

5) Xie F. Y., Woodle M. C., Lu P. Y., Drug Discov. Today, 11, 67-73 (2006).

6) Lu P. Y., Xie F. Y., Woodle M. C., Trends Mol. Med., 11, 104-113 (2005).

7) Hashida M., Kawakami S., Yamashita F., Chem. Pharm. Bull., 53, $871-880$ (2005).

8) Spiess M., Biochemistry, 29, 10009-10018 (1990).

9) Hirabayashi K., Yano J., Inoue T., Yamaguchi T., Tanigawara K., Smyth G. E., Ishiyama K., Ohgi T., Kimura K., Irimura T., Cancer Res., 59, 4325-4333 (1999).

10) Sonoke S., Ueda T., Fujiwara K., Sato Y., Takagaki K., Hirabayashi K., Ohgi T., Yano J., Cancer Res., 68, 8843-8851 (2008).

11) Nilsson M., Berg T., Biochim. Biophys. Acta, 497, 171-182 (1977).

12) Chowdhury N. R., Wu C. H., Wu G. Y., Yerneni P. C., Bommineni V. R., Chowdhury J. R., J. Biol. Chem., 268, 11265-11271 (1993).

13) Sternberg B., Hong K., Zheng W., Papahadjopoulos D., Biochim. Biophys. Acta, 1375, 23-35 (1998).

14) Semple S. C., Akinc A., Chen J., Sandhu A. P., Mui B. L., Cho C. K., Sah D. W. Y., Stebbing D., Crosley E. J., Yaworski E., Hafez I. M., Dorkin J. R., Qin J., Lam K., Rajeev K. G., Wong K. F., Jeffs L. B., Nechev L., Eisenhardt M. L., Jayaraman M., Kazem M., Maier M. A., Srinivasulu M., Weinstein M. J., Chen Q., Alvarez R., Barros S. A., De S., Klimuk S. K., Borland T., Kosovrasti V., Cantley W. L., Tam Y. K., Manoharan M., Ciufolini M. A., Tracy M. A., de Fougerolles A., MacLachlan I., Cullis P. R., Madden T. D., Hope M. J., Nat. Biotechnol., 28, 172-176 (2010).

15) Koltover I., Salditt T., Rädler J. O., Safinya C. R., Science, 281, 7881 (1998).

16) Fadden A. J., Holt O. J., Drickamer K., Glycobiology, 13, 529—537 (2003).

17) Sato A., Takagi M., Shimamoto A., Kawakami S., Hashida M., Biomaterials, 28, 1434-1442 (2007).

18) Wang S. L., Yu F. B., Jiang T. Y., Sun C. S., Wang T., Zhang J. H., J. Drug Target., 16, 233-242 (2008). 